\title{
Implications of unusual pitch-angle distributions observed by ISEE-1 and 2
}

\author{
C. A. Zuluaga, E. S. Beiser, J. Chen, and T. A. Fritz \\ Center for Space Physics, Boston University, Boston MA, USA \\ Received: 14 May 2004 - Revised: 6 October 2006 - Accepted: 13 October 2006 - Published: 22 November 2006
}

\begin{abstract}
Unusual energetic particle pitch angle distributions (PADs) were observed by the ISEE-1 and 2 satellites at $3 \mathrm{~h}$ MLT and a radial distance of about $10-15 R_{E}$ during the time period of 07:00-14:00 UT on 3 March 1979. The ISEE-1 satellite obtained complete 3-D distributions of energetic proton and electron fluxes as a function of energy, while ISEE- 2 was configured to provide higher time resolution but less angular resolution than ISEE-1. The ISEE-1 observed a butterfly PAD (a minimum in the $90^{\circ}$ PA particle flux) for a period of about $2 \mathrm{~h}(10: 00-12: 00 \mathrm{UT})$ for the electrons, and $3 \mathrm{~h}$ (09:00-12:00 UT) for the protons over an energy range of 22.5-189 keV (E1-E4) for the electrons and 24-142 keV (P1-P4) for the protons. The small pitch angle $\left(15^{\circ}, 30^{\circ}\right)$ charged particles (electrons and protons) are seen to behave collectively in all four energy ranges. The relative differences in electron fluxes between $15^{\circ} \mathrm{PA}$ and $90^{\circ} \mathrm{PA}$ are more significant for higher energy channels during the butterfly PAD period. Three different types of electron PADs (butterfly, isotropic, and peaked-at- $90^{\circ}$ ) were observed at the same location and time as a function of energy for a short period of time before 10:00 UT. Electron butterfly distributions were also observed by the ISEE-2 for about $1.5 \mathrm{~h}$ over $28-62 \mathrm{keV}$ (E2-E4), although less well resolved than ISEE-1. Unlike the ISEE-1, no butterfly distributions were resolved in the ISEE-2 proton PADs due to less angular resolution. The measured drift effects by ISEE-1 suggest that the detected protons were much closer to the particle source than the electrons along their trajectories, and thus ruled out a nightside source within 18:00 MLT to 03:00 MLT. Compared to 07:30 UT, the charged particle fluxes measured by ISEE-1 were enhanced by up to three orders of magnitude during the period 08:30-12:00 UT. From 09:10:00 UT to 11:50 UT, the geomagnetic conditions were quiet $(\mathrm{AE}<100 \mathrm{nT})$, the LANL geosynchronous satellites observed no substorms, and the lo-
\end{abstract}

Correspondence to: T. A. Fritz

(fritz@bu.edu) cal magnetic field measured by ISEE-1 was almost constant, while the small PA charged particle (both electron and proton) fluxes measured by ISEE-1 increased gradually, which implies a particle source other than the substorm source. Based on detailed particle trajectory tracings in a realistic geomagnetic field model, the $50-200 \mathrm{keV}$ protons with small PA at 10:00 UT ISEE-1 location on 3 March 1979 were passing through the northern high-altitude and high-latitude morningside region where the cusp should be located under a dawnward IMF component condition, while those protons with large PA may connect to the high-latitude morningside magnetopause. It is possible that the cusp source is responsible for the all particles observed during the event.

Keywords. Magnetospheric physics (Magnetotail; Energetic particles, trapped). Space plasma physics (Charge particle motion and acceleration)

\section{Introduction}

The particle pitch angle distributions (PADs) in different regions of the Earth's magnetosphere have been extensively studied and reported. West (1965) made the earliest observations of butterfly PADs. These butterfly distributions were caused by the Russian high-altitude nuclear detonation on 28 October 1962. Various reasons for the natural occurrence of butterfly distributions have been given in literature; such as drift shell splitting (Roederer, 1967, 1970) and either magnetopause shadowing (West et al., 1972, 1973) or a negative radial flux gradient (Pfitzer et al., 1969; Sibeck et al., 1987).

Drift shell splitting arises from the local time asymmetry of the geomagnetic field. Due to the compression by the solar wind the geomagnetic field is not an exact dipole. A magnetic field line for example crossing the equator at $7 R_{E}$ on the dayside will have a larger value of $\mathrm{B}_{E q}$ than a magnetic field line located at the same radial distance on the nightside.

Published by Copernicus GmbH on behalf of the European Geosciences Union. 

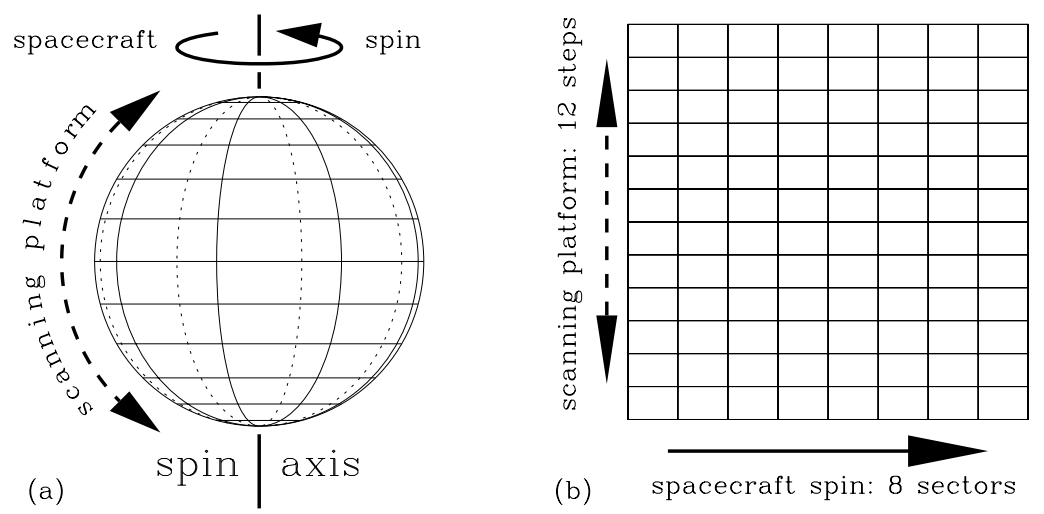

Fig. 1. (a) The measurement of particle fluxes over the unit sphere is produced during one $36.5 \mathrm{~s}$ spin/scan cycle of the WAPS instrument onboard ISEE-1. (b) The unit sphere can be made into a mercator projection, on which pitch angle contours and flux intensities are overlaid. The resulting plot displays the Pitch Angle Distribution (PAD) used in subsequent figures (from Eccles and Fritz, 2002).

Since particles mirroring at the equator and conserving their first adiabatic invariant will follow contours of constant $\mathrm{B}$, a particle that crosses the noon meridian at $X=R_{0}$ will cross the midnight meridian at a distance smaller than $\mathrm{R}_{0}$. Reeves et al. (1991) used the Tsyganenko magnetic field model (Tsyganenko et al., 1982) to show that the drift shells of ions starting at $6.7 R_{E}$ on the noon meridian split to a radial distance range of $6.2 R_{E}$ to $7.4 R_{E}$ on the nightside. Magnetopause shadowing is the process whereby $90^{\circ}$ pitch angle (PA) particles of all energies drift to a larger radial distance than smaller PA particles on the dayside and are lost from the distribution due to scattering at the magnetopause. The loss of these $90^{\circ} \mathrm{PA}$ particles is what we currently refer to as a butterfly PAD. Because the particles arrive at different radial distances on the nightside depending on their equatorial PA, the variation of the particle intensity as a function of radial distance on the dayside will be reflected in the redistribution of these particles on the nightside. Since the intensity of the particle fluxes usually decreases with radial distance at $\mathrm{R}>5 R_{E}$, a minimum in the local nightside $\mathrm{PAD}$ at equatorial pitch angles near $90^{\circ}$ can be produced without the actual loss of any particles.

West et al. (1973) showed that electrons with energies of about $79 \mathrm{keV}$, and at times up to $158 \mathrm{keV}$, could have an isotropic distribution, while higher energy electrons showed a butterfly distribution. Smets et al. (1999) showed that there are three distinct domains due to the magnetic field evolution in the near-Earth tail; each region being most efficient at producing only a single type of distribution. Data from ISEE-1 (International Sun Earth Explorer-1) have shown that butterfly distributions are very common particularly in energetic electrons and are observed for periods of hours in the Earth's magnetotail (Fritz and Chen, 1999; Fritz et al., 2003a). ISEE-1 also observed different types of charged particle PADs at the same time and location as a function of energy (Fritz et al., 2003a).

\section{Instrumentation}

The data set used is mainly from the ISEE-1 spacecraft that was launched together with ISEE-2 on 22 October 1977 into an elliptic orbit with apogee of $23 R_{E}$ and perigee $280 \mathrm{~km}$, a period of about $57 \mathrm{~h}$ and an inclination of about $28^{\circ}$. A variable distance separated the two spacecraft, so data from complementary instruments can be used to separate space/time ambiguities. The Medium Energy Particles Experiment (MEPE) sensors on each satellite were designed to detect electrons and ions ranging from $22.5 \mathrm{keV}$ to $1.2 \mathrm{MeV}$ for electrons and $24 \mathrm{keV}$ to $2 \mathrm{MeV}$ for protons. For the WIM instrument aboard ISEE-1 these energy ranges were divided into eight energy channels each for electrons and ions in the standard Low Bit Rate [LBR] mode. The WAPS (Wide Angle Particle Spectrometer) on ISEE-1 was mounted on a scan platform which rotated $160^{\circ}$ from nearly parallel to nearly anti-parallel to the spin axis in 12 satellite spin periods which required about $36.5 \mathrm{~s}$. The sensor had a look direction opening conical half-angle of 5.3 ${ }^{\circ}$. Williams et al. (1978) have given a detailed description of this instrument. The data returned from the ISEE-1 MEPE were converted to flux for each of the energy passbands and plotted as a function of both time and L-value (McIlwain, 1961) for the two years of operational life of the ISEE-1 experiment. Using simultaneous measurements of the geomagnetic field provided by the onboard magnetometer (Russell, 1978), the instantaneous pitch angle of a given particle measurement was determined. As illustrated in Fig. 1 (after Eccles and Fritz, 2002) for each $36.5 \mathrm{~s} \mathrm{spin/scan} \mathrm{cycle} \mathrm{of} \mathrm{the} \mathrm{WAPS,} \mathrm{a} \mathrm{repre-}$ sentative flux corresponding to each energy channel for eight sectors [LBR mode] of each spin was determined. This resulted in 96 samples being made on the $4 \pi$ steradian unit sphere. The spin axes of the ISEE satellites were essentially perpendicular to the ecliptic plane. These fluxes could be displayed in a color coded manner on a mercator projection of the unit sphere where the vertical axis is the polar angle 
and the horizontal axis is the azimuthal angle in spacecraft coordinates which were essentially Geocentric Solar Ecliptic [GSE] coordinates. The scale on the horizontal axis $[\varphi]$ has the sunward direction at $0^{\circ}$ and the antisunward direction at $180^{\circ}$. For clarity it is emphasized that the look direction is the direction from which the energetic particles are coming. The vertical axis direction $[\theta]$ is the scan direction where $\theta=0^{\circ}$ is antiparallel to the spacecraft spin axis viewing the south ecliptic pole and $\theta=180^{\circ}$ is parallel to the spin axis viewing the north ecliptic pole. At $\theta=90^{\circ}$, the $0^{\circ}$ and $90^{\circ}$ azimuthal directions represent essentially the GSE X- and Y-axes, respectively. The pitch angle associated with each flux measurement was determined instantaneously from the magnetometer data and used to place contours of constant pitch angle on these unit sphere angle/angle $[\theta, \varphi]$ displays (from Fritz et al., 2003a).

Aboard the ISEE-2 spacecraft was the KED instrument. The KED instrument consisted of a WAPS sensor plus four additional Narrow Angle Particle Spectrometers (NAPS) mounted in fixed positions with respect to the spin axis on the ISEE-2 satellite. A drawing of the KED instrument is presented in Fig. 2. The WAPS on ISEE-2 functioned in a similar manner to the one mounted on ISEE-1. The NAPS used a homogenous magnetic field to separate the electrons from the ions. The ions traversed the field unaffected and were detected similar to the manner in which they were detected by the WAPS. The KED instrument provided a higher time resolution, but less detailed angular resolution with the multiple sensors. ISEE-2 had two operational modes, mode A and mode $\mathrm{B}$, which maintained the same data format (Williams et al., 1978). In mode A, all 5 sensors contributed equal fractions of the data stream. Options were possible to give the ions or electrons full rate, or both ions and electrons in a given direction. In mode B, most of the data stream was devoted to the WAPS (no significant contribution was received from the NAPS). For each spin the detailed energy channel data was either from a single ion or electron detector.

\section{Observations}

From 07:00 UT to 14:00 UT on 3 March 1979 the satellites ISEE-1 and 2 were moving inbound in the nightside magnetotail of the magnetosphere at a location of about 03:00 MLT with a radial distance of about $14 R_{E}$. Figure 3 shows the projection of the ISEE satellites trajectories onto the GSM XY plane (left panel) and GSM XZ plane (right panel) for this time period when the satellites were close to the equatorial plane (-1.6>GSM Z $>-2.7 R_{E}$ ) (right panel of Fig. 3).

The top panel of Fig. 4 plots the time profiles of the electron fluxes measured by ISEE- 1 during this time period. Four different pitch angles $\left(15^{\circ} \pm 5^{\circ}, 30^{\circ} \pm 5^{\circ}, 60^{\circ} \pm 5^{\circ}\right.$, $90^{\circ} \pm 5^{\circ}$ ) are plotted for each of the four energy levels: E1 $(22.5-39 \mathrm{keV})$, E2 $(39-75 \mathrm{keV})$, E3 $(75-120 \mathrm{keV})$, E4 $(120-189 \mathrm{keV})$. All of the different energies follow the

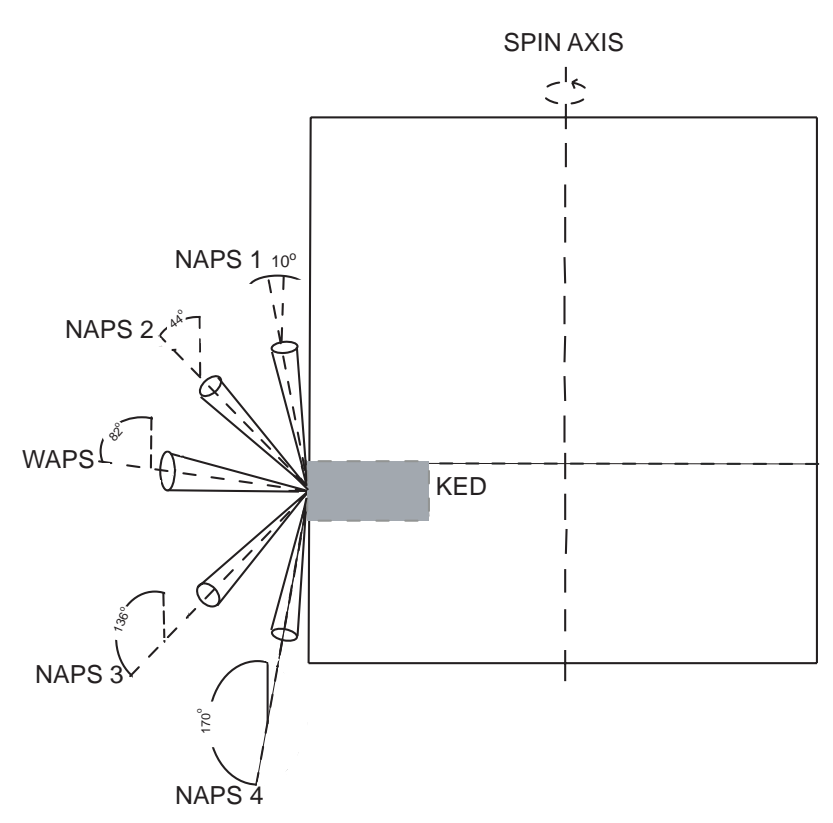

Fig. 2. The scanning ranges of the KED instrument onboard the ISEE-2 satellite. The contribution of each detector depends on the operational mode of the satellite.

same flux scale on the y-axis, but the fluxes of each energy channel are offset by a power of ten in order to fit all energy channels into one plot. In this figure, we observe an isotropic distribution in all energy ranges up to about a time of 09:10 UT. After this time, only the small PA $\left(15^{\circ}\right.$ and $\left.30^{\circ}\right)$ electrons appear to behave in an similar manner in all energy ranges aside from a small flux difference most notably observed in channels E2 and E3 during the time of 10:0011:00 UT. From about 09:10-9:15 UT, the E3 and E4 large PA $\left(60^{\circ}\right.$ and $\left.90^{\circ}\right)$ electron fluxes start to vary differently with respect to the small PA electron flux. The large PA electron fluxes in E2, E3, and E4 are seen to have sharp increases around 09:25 UT and 09:50 UT. A slight variation in the large PA electrons from the small PA electrons is seen in energy energy channel E1, but not to the extent observe in the other three energy channels. At around 10:00 UT, the large PA electron fluxes dropped over an order of magnitude in the E3 and $\mathrm{E} 4$ channels, and a little less than an order of magnitude in the E2 channel. From 10:00 UT to 12:00 UT, while the $60^{\circ}$ and $90^{\circ} \mathrm{PA}$ electrons seem to act as one group in energy channel E1 and E2, where close to a constant flux is maintained, the large PA electrons are seen to behave differently in channels E3 and E4. At about 10:10 UT in E3 and E4, the $60^{\circ} \mathrm{PA}$ electron fluxes start to gradually increase until about 12:05 UT when they match in intensity to the small PA electrons; however, the $90^{\circ} \mathrm{PA}$ electron fluxes remain low and recover very quickly at about 12:00 UT. The sharp increase in $90^{\circ} \mathrm{PA}$ electron fluxes is also observed in channels E1 and E2 at about 12:10 UT and 12:05 UT, respectively, although not as pronounced as in E3 and E4. During the two hour 
ISEE 1 \& 2 on 3 March 1979 (062), 7-14 UT
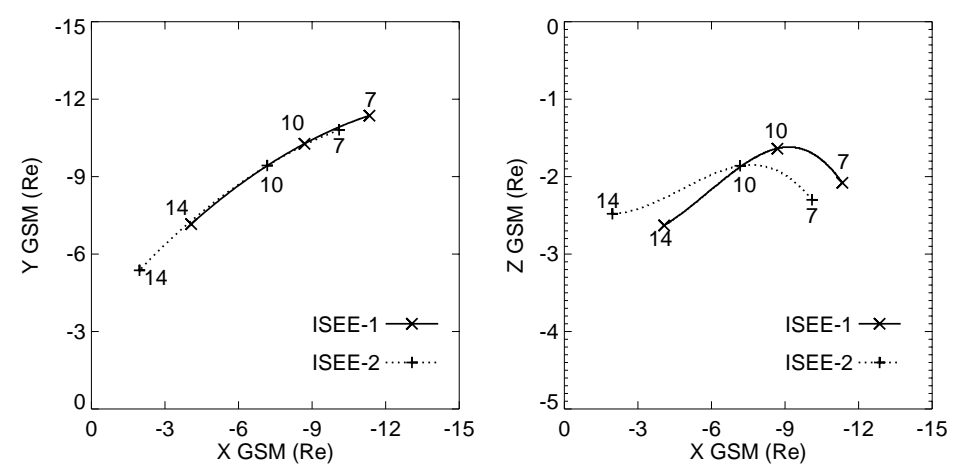

Fig. 3. Orbits of ISEE-1 and 2 projected onto the GSM XY plane (left panel) and XZ plane (right panel) during the time period of 07:00 to 14:00 UT on 3 March 1979 (day 062).

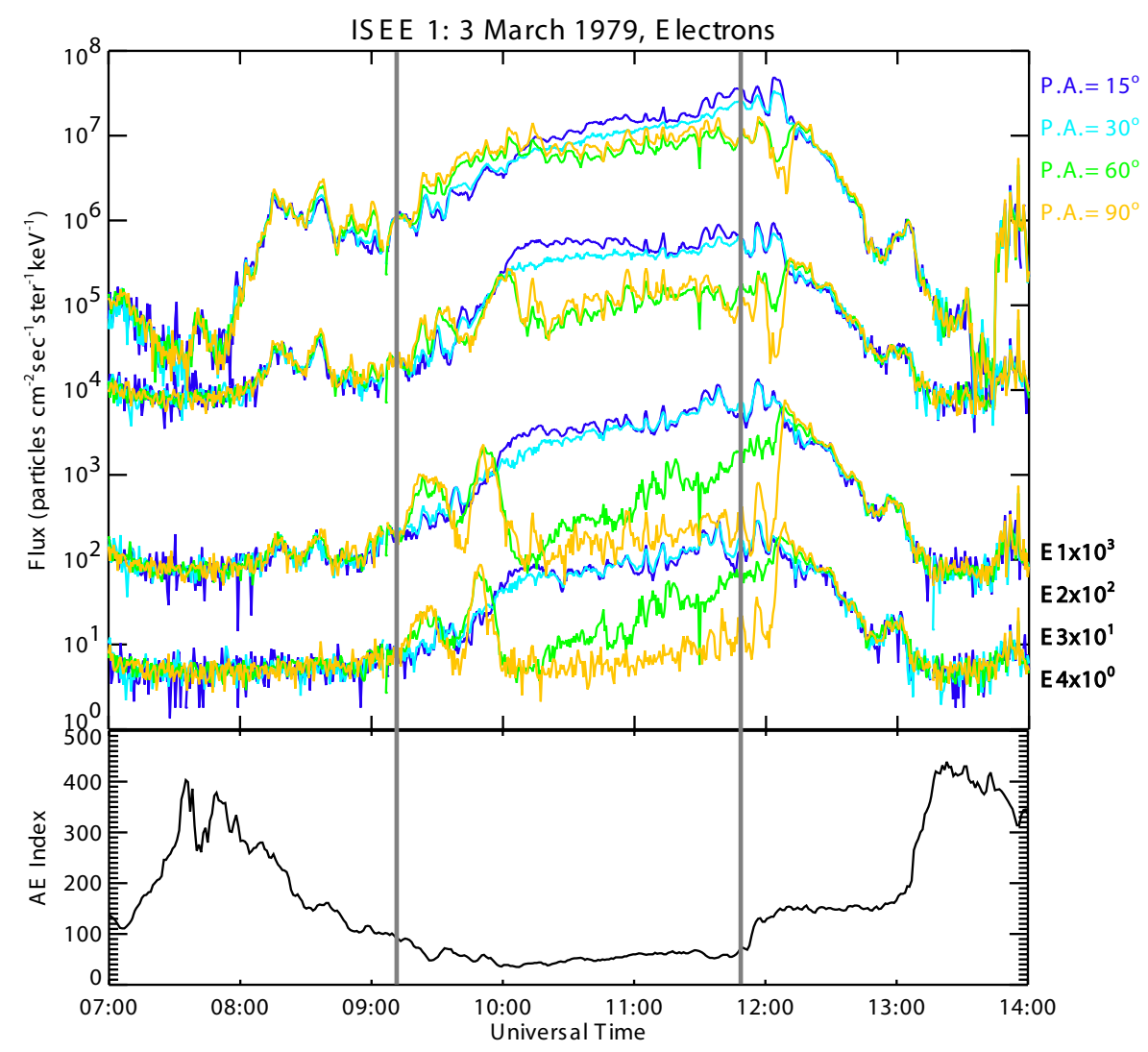

Fig. 4. Top panel displays the pitch angle flux distributions for electron channels E1 (22.5-39 keV), E2 (39-75 keV), E3 (75-120 keV), E4 (120-189 keV) observed by ISEE-1 for the time period of 07:00-14:00 UT. Pitch angles of $15^{\circ} \pm 5^{\circ}, 30^{\circ} \pm 5^{\circ}, 60^{\circ} \pm 5^{\circ}, 90^{\circ} \pm 5^{\circ}$ were sampled. Bottom panel is the AE index vs. time.

period of 10:00-12:00 UT, the butterfly distributions appear most apparent in channels E2, E3, and E4, where the flux of the small PA electrons is seen to be almost an order of magnitude greater than that of the $90^{\circ} \mathrm{PA}$ electrons. The difference between the large and small PA electron fluxes is not as great in channel E1, but it is still responsible for the butter- flies observed in E1. After 12:15 UT, the electron fluxes then proceeded to isotropically decrease by more than two orders of magnitude for all energy channels. 


\section{ISEE 1 Electrons 3 March 1979}

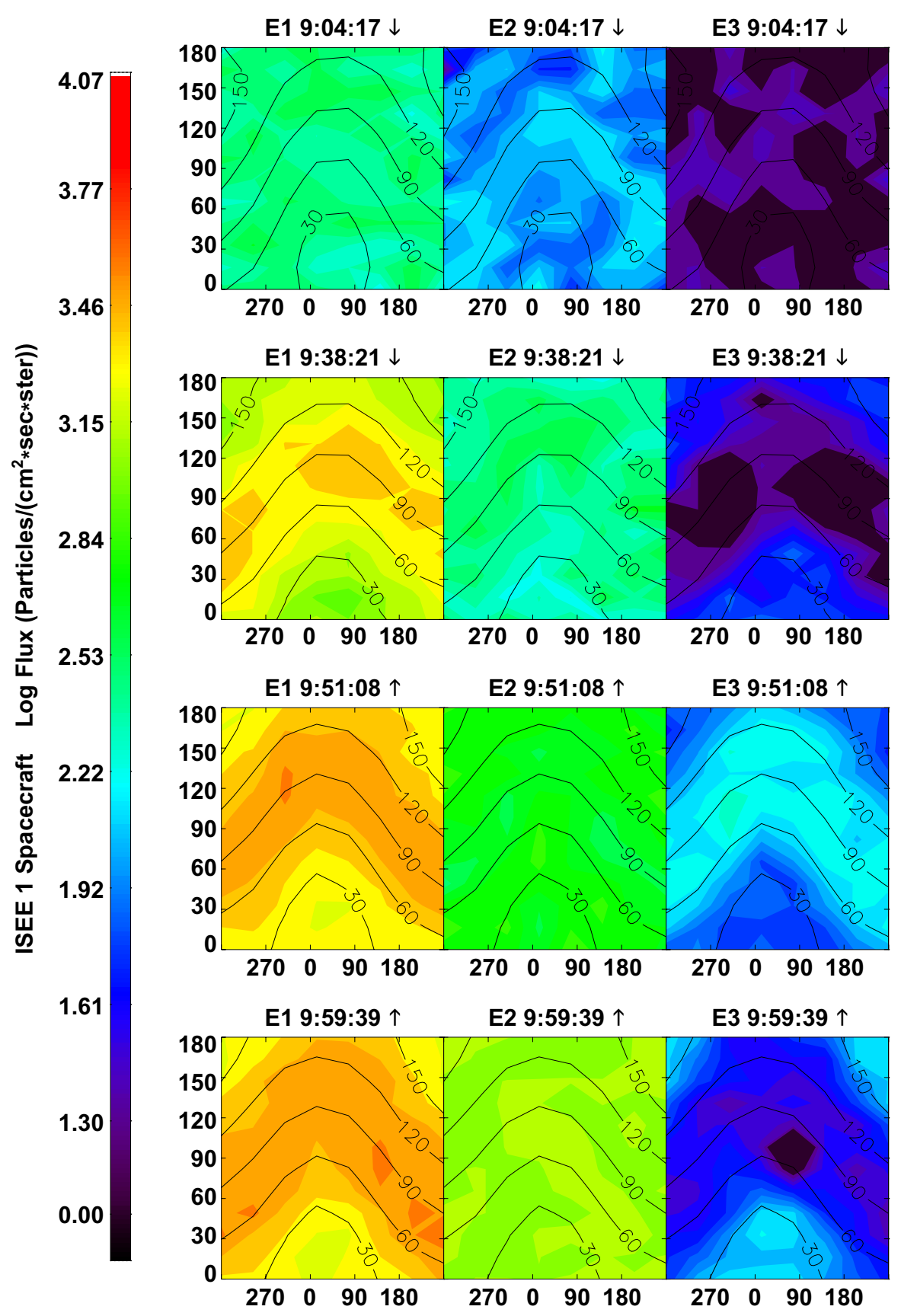

Fig. 5. The 3-D electron PADs for channels E1, E2, and E3 (three columns) at four different times (four rows for 09:04:17, 09:38:21, 09:51:08, and 09:59:39 UT, respectively).

The unusual PADs are more evident in Fig. 5 that shows the electron PADs for channels E1, E2, and E3 at four different times within the period of 09:04-10 UT. These 3-D data are plotted in the mercator projection of the unit sphere scanned in $36.5 \mathrm{~s}$ by ISEE-1 and illustrated in Fig. 1. The three columns represent three different energy channels (E1,
E2, and E3), while the four rows represent four different indicated times. An isotropic distribution in all three energy channels was observed at 09:04:17 UT (the first row of Fig. 5). Later at 09:38:21 UT, each channel had a different PAD; that is, while the E2 channel remained isotropic, an enhancement of the electron intensity around the $90^{\circ} \mathrm{PA}$ 


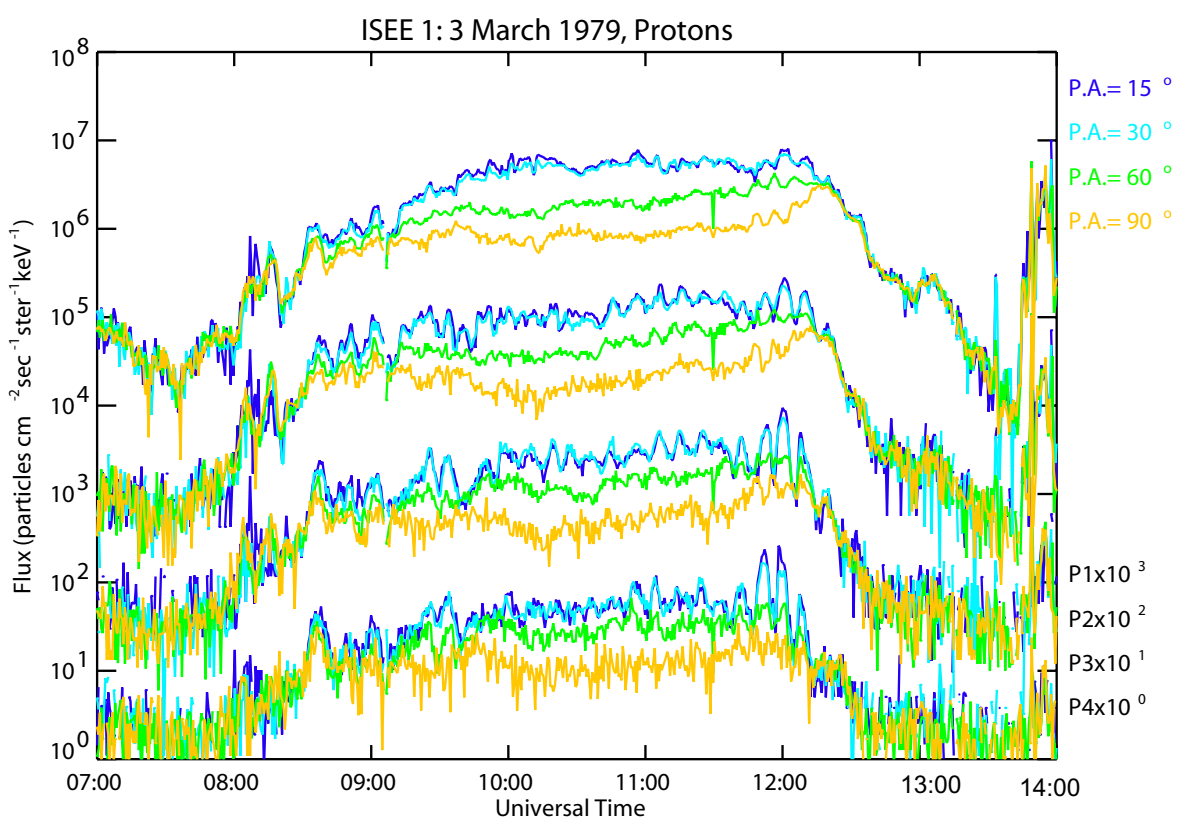

Fig. 6. Pitch angle flux distributions for protons in the energy channels P1 (24.0-44.5 keV), P2 (44.5-65.3 keV), P3 (65.3-95.5 keV), P4 (95.5-142.0 keV) recorded by ISEE-1. Pitch angles of $15^{\circ} \pm 5^{\circ}, 30^{\circ} \pm 5^{\circ}, 60^{\circ} \pm 5^{\circ}$, and $90^{\circ} \pm 5^{\circ}$ are displayed.

in channel E1 and a butterfly distribution in channel E3 were observed. At 09:51:08 UT (the third row), only two different distributions: a peaked-at- $90^{\circ}$ in channels $\mathrm{E} 1$ and $\mathrm{E} 3$, and still an isotropic distribution in channel E2 were measured. About eight minutes later at 09:59:39 UT, the three different distributions seen in row two were seen in row four but with much wider butterfly and peaked-at- $90^{\circ}$ distributions.

Similar to Fig. 4, Fig. 6 shows the ISEE-1 proton fluxes vs time for four different PAs at 07:00-14:00 UT. Here the butterfly distributions were again observed for an extended period but they were not caused by a sharp decrease of near perpendicular PA fluxes as in the case for the fluxes of the electrons at around 10:00 UT. Small and large PA protons seem to behave isotropically up to about 08:30 UT. At around 08:40 UT, an hour earlier than it was observed in the electron flux distributions, a slightly higher intensity was seen for the $15^{\circ}$ and $30^{\circ} \mathrm{PA}$ protons than the $60^{\circ}$ and $90^{\circ} \mathrm{PA}$ ones. The protons seem to behave as three different groups during the time of interest. The $15^{\circ}$ and $30^{\circ}$ PA protons behave as one group in all four energy channels with the largest intensities, while the $90^{\circ}$ PA protons have the lowest intensitiies and the $60^{\circ}$ PA protons were in between. These variations in the behavior of the small and large PA protons produce a butterfly distribution in all four energy channels that persists for a period of about $3 \mathrm{~h}$ (09:00-12:00 UT). Unlike the sharp increase in the $90^{\circ} \mathrm{PA}$ electron fluxes at around 12:05 UT, a steady increase in the flux for these large PA protons brings the distribution back to isotropic at around 12:15 UT.

Similar to Fig. 5, Fig. 7 shows an angle/angle plot for four different times (10:01:28, 10:41:01, 11:20:33, and
11:52:11 UT) for P1, P2, P3, and P4. A clear butterfly PAD with a minimum proton intensity at $90^{\circ} \mathrm{PA}$ can be seen in all four proton energy channels.

ISEE-2 data demonstrating the butterfly PADs is presented in Fig. 8 which shows the electron flux distributions throughout the time period for 2 different pitch angles: $90^{\circ} \pm 5^{\circ}$ and $40^{\circ} \pm 5^{\circ}$. Due to the operational mode of the instrument during the time period studied, data for small PA particles were not available. The butterfly distributions were seen to appear around 11:00 UT, one hour later from when they were observed by the ISEE-1 satellite. The butterfly distributions in this case were produced similar to those observed in the protons seen by ISEE-1. Rather than a sharp decrease in near perpendicular PA particles, the flux in the $40^{\circ} \mathrm{PA}$ electrons was seen to continue to increase after a leveling off in the $90^{\circ}$ PA electron flux. Similar to the ISEE-1 electron PADs, the distribution returned to isotropic around 12:00 UT, and continued to isotropically decrease for over two orders of magnitude. The proton PADs showed no butterflies in the data collected by ISEE-2 (Fig. 9) but this could be due to a limitation of the ISEE-2 WAPS sensor to observe small pitch angles.

Figure 10 shows the magnetic field values recorded by each satellite (solid lines) and modeled by the T04 (Tsyganenko and Fairfield, 2004) magnetic field model (dashed lines) during the time interval of interest. It is observed that no significant variations happened in the total magnetic field $\left(\mathrm{B}_{\mathrm{T}}\right)$ during 07:00-12:00 UT. 


\section{ISEE 1 Ions 3 March 1979}

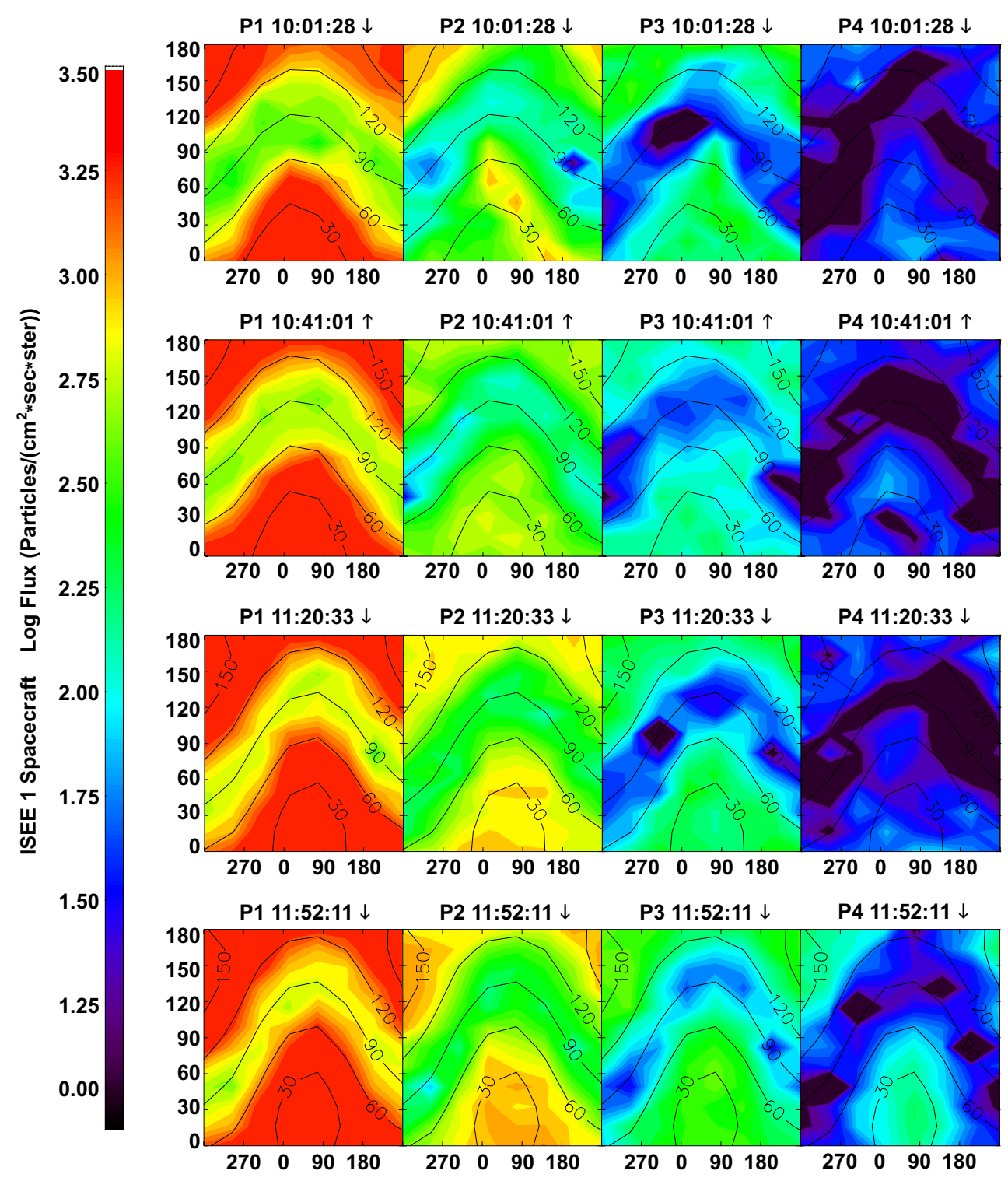

Fig. 7. The 3-D proton PADs for channels P1, P2, P3, and P4 (four columns) at four different times (four rows for 10:01:28, 10:41:01, 11:20:33, and 11:52:11 UT, respectively).

\section{Trajectory generator}

By integrating Newton's second law with the Lorentz force using a code developed at Boston University by Sullivan (2002) known as TRAJGEN, one can obtain the trajectory of a charged particle in the presence of an electromagnetic field. When integrating the equations of motion, the magnetic and electric field values for the specific points are required. In the TRAJGEN program, currently the electric field can be modeled either as a uniform (possibly null) field or as the planar E3 model proposed by Mcllwain (1974). The mag- netic field models available are the planar M2 model also proposed by Mcllwain (1974), a tilted-enhanced-image dipole, and the T96 model of Tsyganenko (1996). The TRAJGEN has been used before for $200 \mathrm{keV}$ proton tracing (Fritz et al., 2000), showing a connection between the nightside plasma sheet and the dayside high-altitude cusp by the proton drift path.

From about 09:10:00 UT to 11:50 UT (between two vertical lines in Fig. 4) on 3 March 1979, the AE index was less than $100 \mathrm{nT}$ (bottom panel of Fig. 4), indicating a geomagnetically quiet period. Previously, Kosik (1979) had 


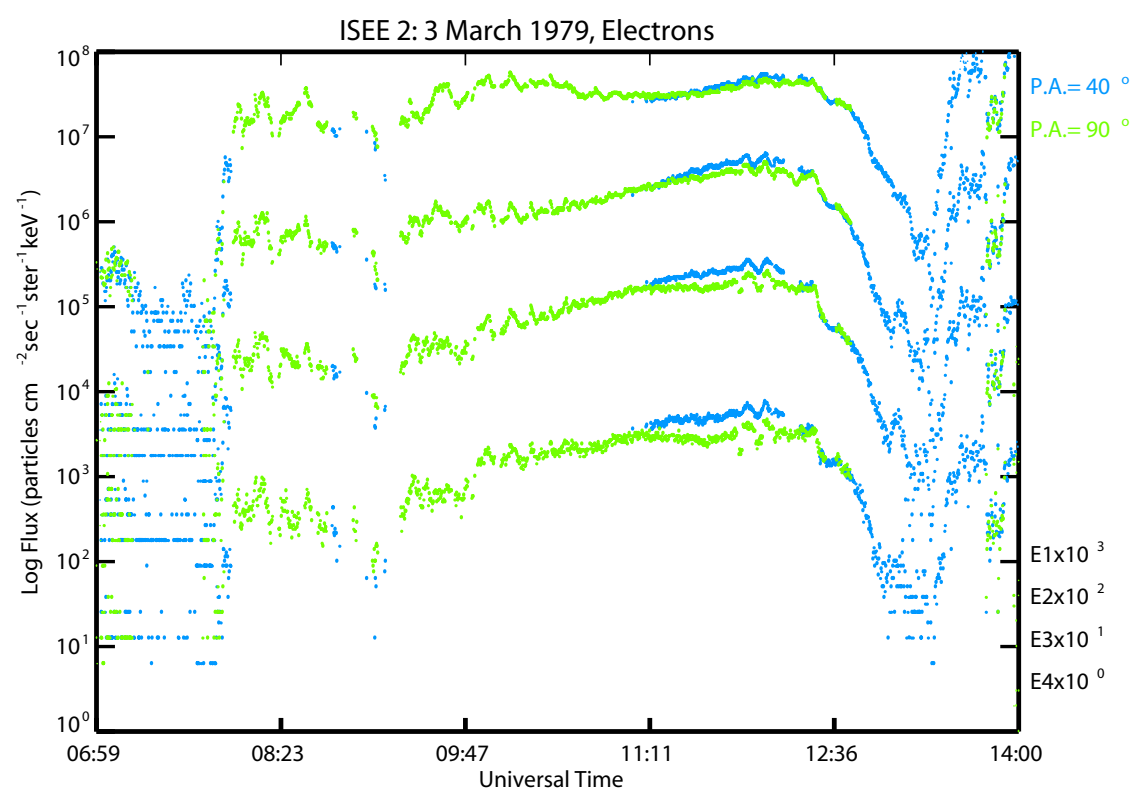

Fig. 8. ISEE-2 electron intensity as a function of time in the channels E1 (17.5-28 keV), E2 (28-38 keV), E3 (38-48 keV), and E4 (48$62 \mathrm{keV}$ ) observed at 07:00-14:00 UT on 3 March 1979. The pitch angles displayed are $40^{\circ} \pm 5^{\circ}$ and $90^{\circ} \pm 5^{\circ}$.

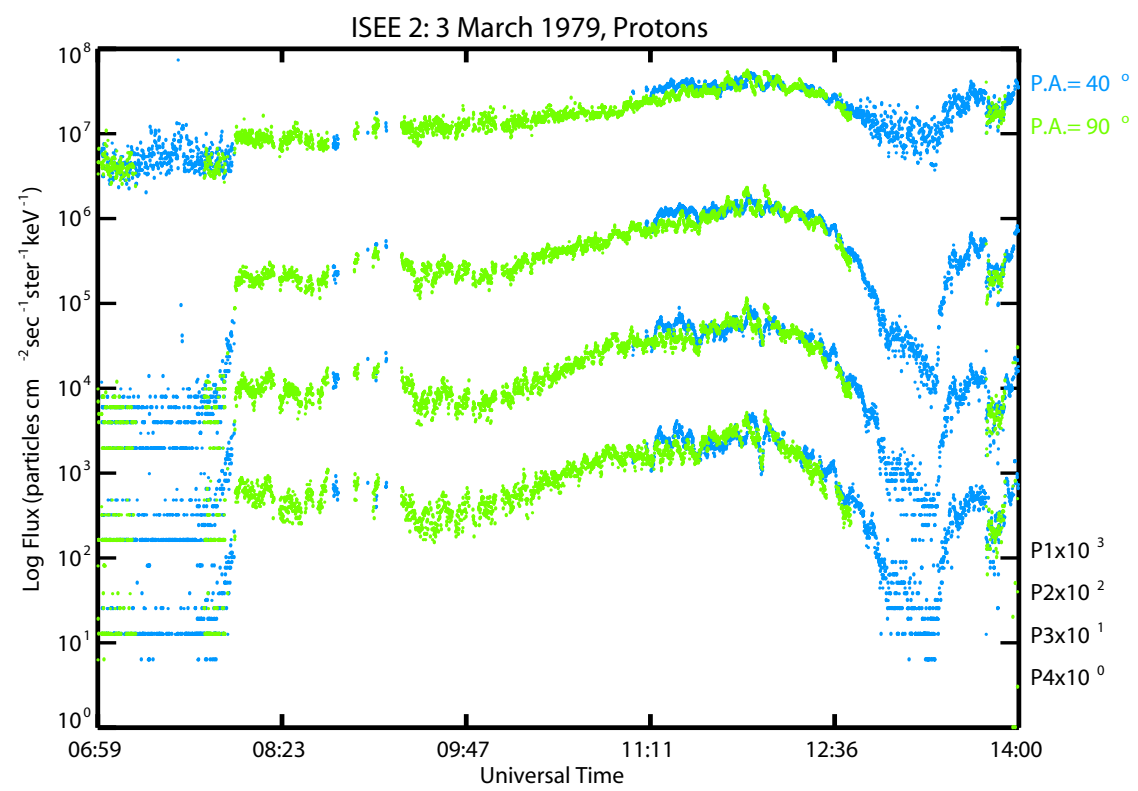

Fig. 9. ISEE-2 proton intensity as a function of time in the channels P1 (17.5-28 keV), P2 (28-38 keV), P3 (38-48 keV), and P4 (48-62 keV) observed at 07:00-14:00 UT on 3 March 1979. The pitch angles displayed are $40^{\circ} \pm 5^{\circ}$ and $90^{\circ} \pm 5^{\circ}$.

reported that during a magnetically quiet period the convection electric field is very weak and the magnetic field asymmetry produces the dominant effect. Sibeck et al. (1987) further showed that compared to gradient and curvature drifts the electric drifts are negligible for the $>25 \mathrm{keV}$ particles. Therefore, for the present particle trajectory traces, an $\mathrm{E}=0$ electric field was assumed along with the T96 model. The main use of the TRAJGEN is for tracing particle trajectories forward or backward in time. The momentum of the particle is strictly conserved. For tracing backward one must simply give an electron an initial positive charge, and this will cause the electron to drift west rather than east. Similarly with the proton, a negative charge will cause it to drift east rather than west. Figure 11 shows a sample run, in which 


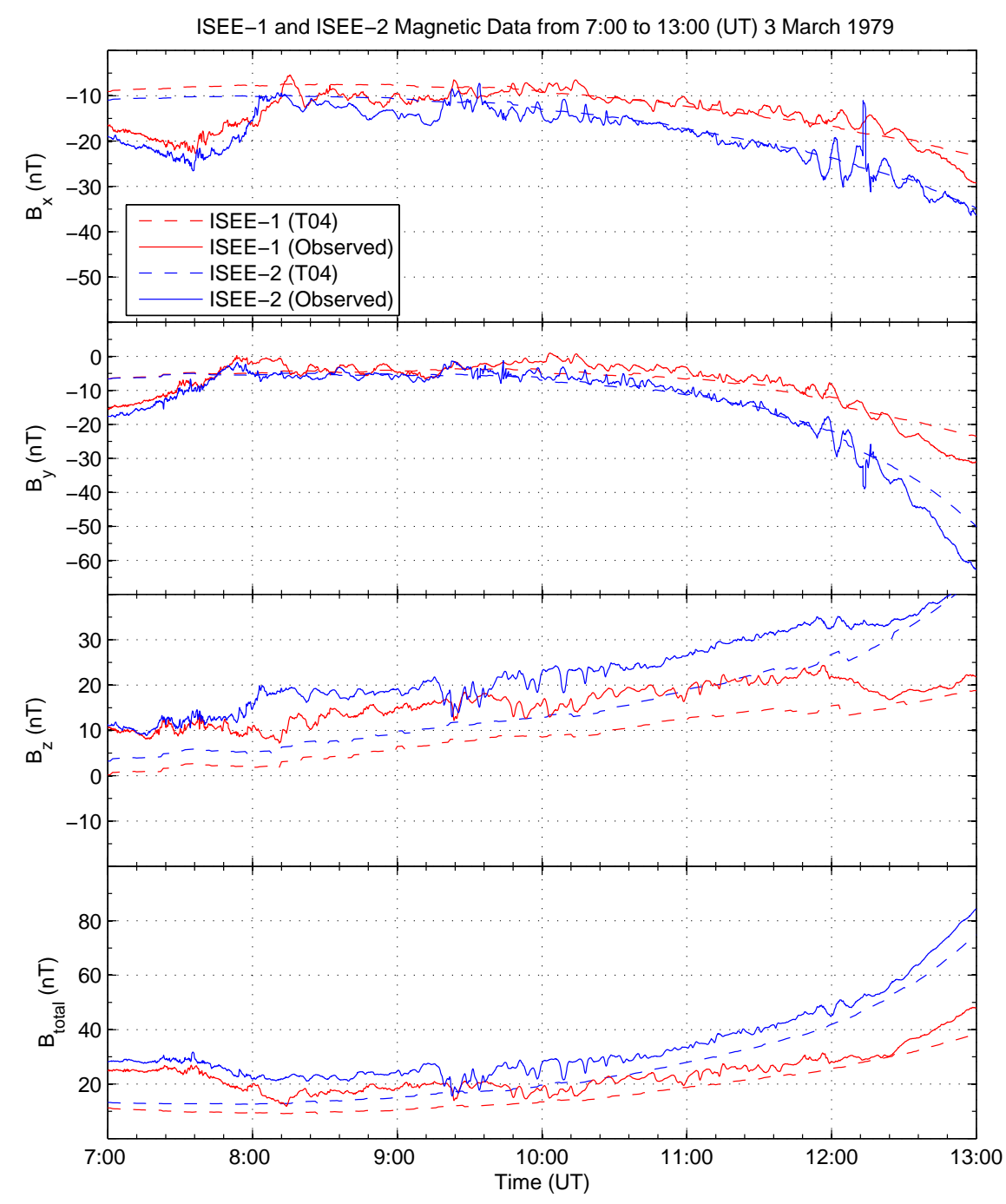

Fig. 10. Local magnetic field $\left(\mathrm{B}_{\mathrm{X}}, \mathrm{B}_{\mathrm{Y}}, \mathrm{B}_{\mathrm{Z}}\right.$, and $\mathrm{B}_{\mathrm{T}}$ ) data recorded by the magnetometer onboard ISEE-1 (red solid line) and ISEE-2 (blue solid line) and modeled by the T04 geomagnetic field model at ISEE-1 (red dashed line) and ISEE-2 (blue dashed line) locations during 07:00-13:00 UT on 3 March 1979.

a proton was started at the dayside equator $5 R_{E}$ from the Earth and was given a negative charge to trace the particle to its source. The date of 3 March 1979 was picked and the required parameters were obtained for the T96 model. The right bottom provides the information entered for the run. In the first plot of the XZ coordinates, the bounce motion of the particle can be observed. This bounce motion characteristic of a particle's trajectory is better observed in the second (YZ) plot, where a bounce motion as well as a drift motion around the Earth can be clearly seen. The drift however is better seen on the third plot in the XY plane with the Sun in the positive $\mathrm{X}$ direction (GSM coordinates). The particle is clearly seen to have drifted westward in order to arrive at its observed destination. The fourth panel shows the particle's location off of the equatorial plane as a function of time. This provides us with a look at the bounce motion as a function of time. Figure 12 provides more insight from the selected run. Panel 1 (top left) shows the pitch angle of the particle as a function of time. As the particle bounces between the mirror points, the particle's pitch angle varies from its max and min values alternately as it crosses the equator. The right panel shows a close up of this plot. The middle panel shows the particle's first adiabatic invariant as a function of time, a quantity that should remain constant, and finally the bottom panel shows the absolute value of the magnetic field along the trajectory where the maximum magnetic field associated with the mirror point should remain constant as it appears to do in the panel. This example of a $100 \mathrm{keV}$ proton drifting completely around the Earth returning to its origin at $5 R_{E}$ in 60 min illustrates the capability of the TRAJGEN code and the lack of a build-up of errors. 

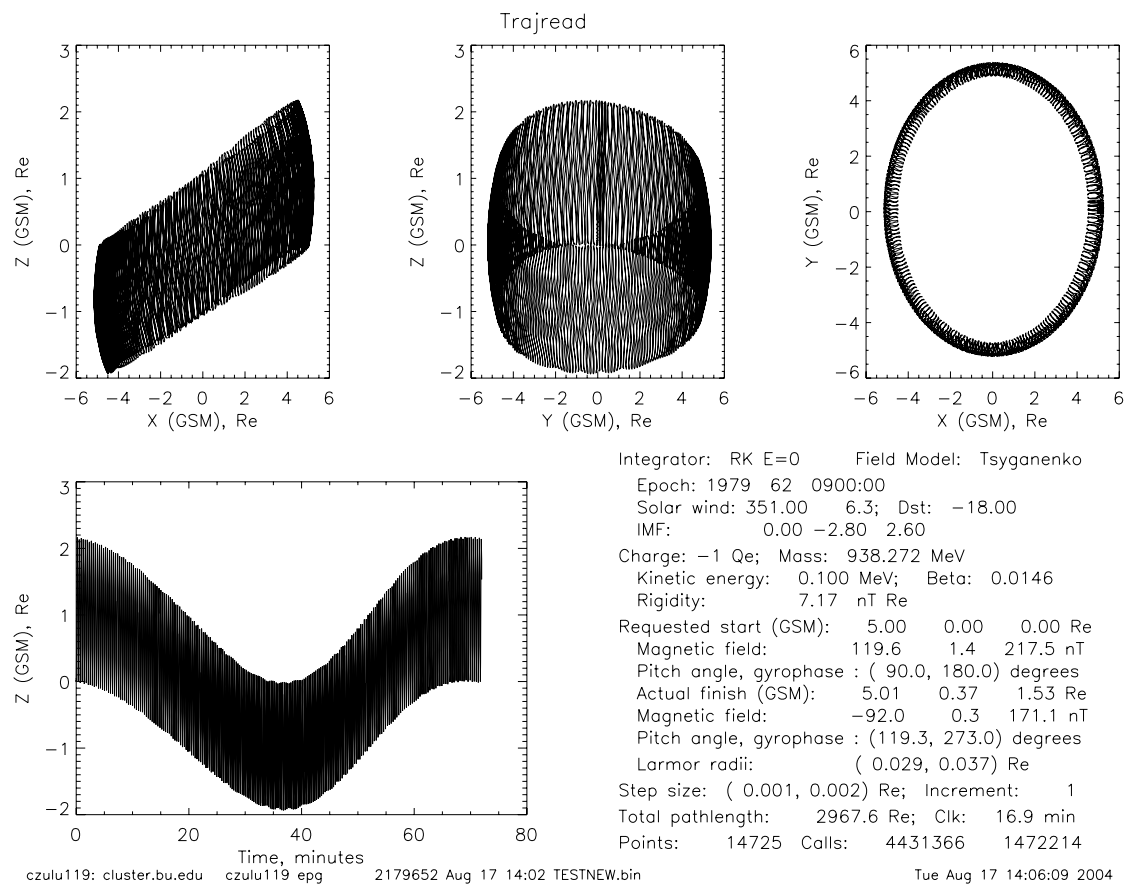

Fig. 11. Example of TRAJGEN code proton trace-back. The trajectory of the particle is plotted in the GSM (a) X-Z (b) Y-Z (c) X-Y planes as well as (d) $\mathrm{Z}$ vs. time. The text box provides information about the run.
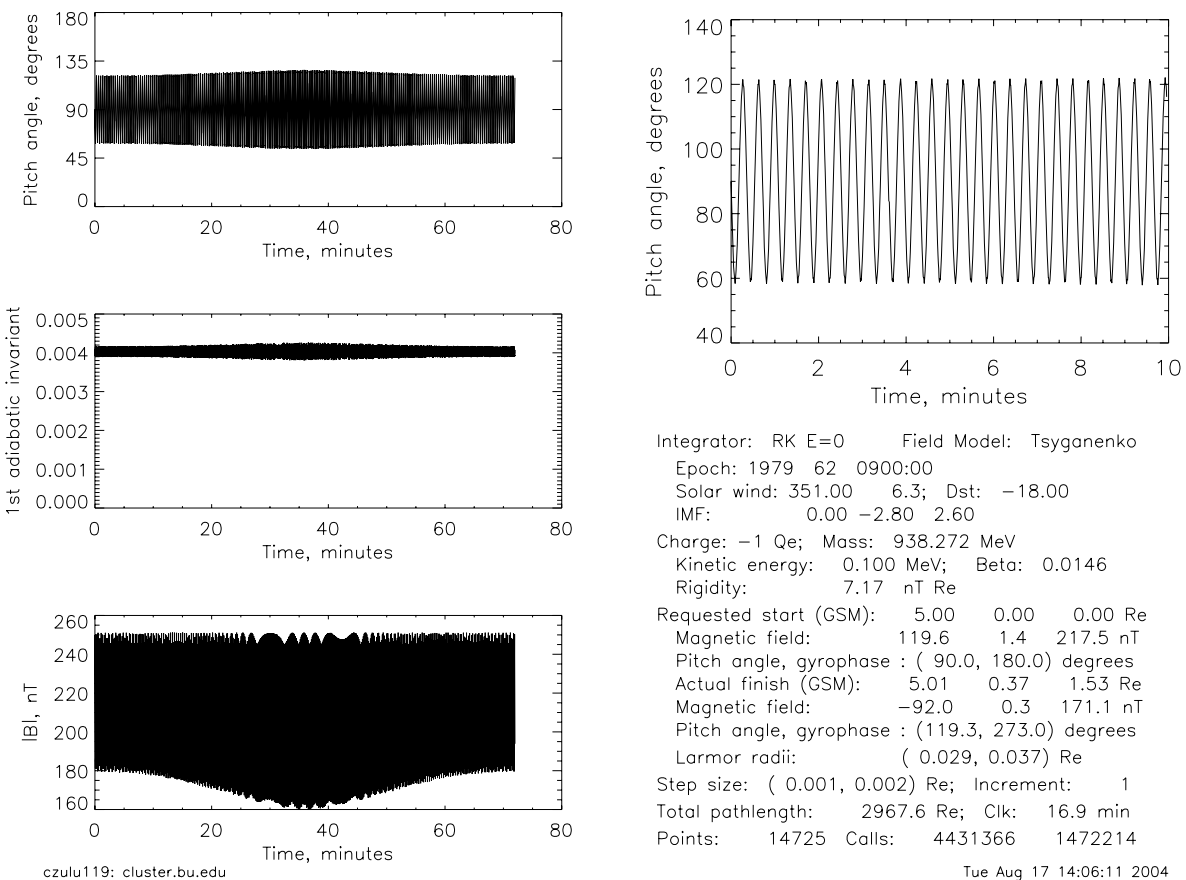

Tue Aug 17 14:06:11 2004

Fig. 12. Continued example of TRAJGEN run for the proton particle of Fig. 11. The pitch angle of the particle and its first adiabatic invariant are both plotted as a function of time. The magnitude of the magnetic field (as calculated using the T96 model) is also plotted versus time. A close up of the pitch angle of the particle versus time for the first $12 \mathrm{~min}$ is also plotted. 


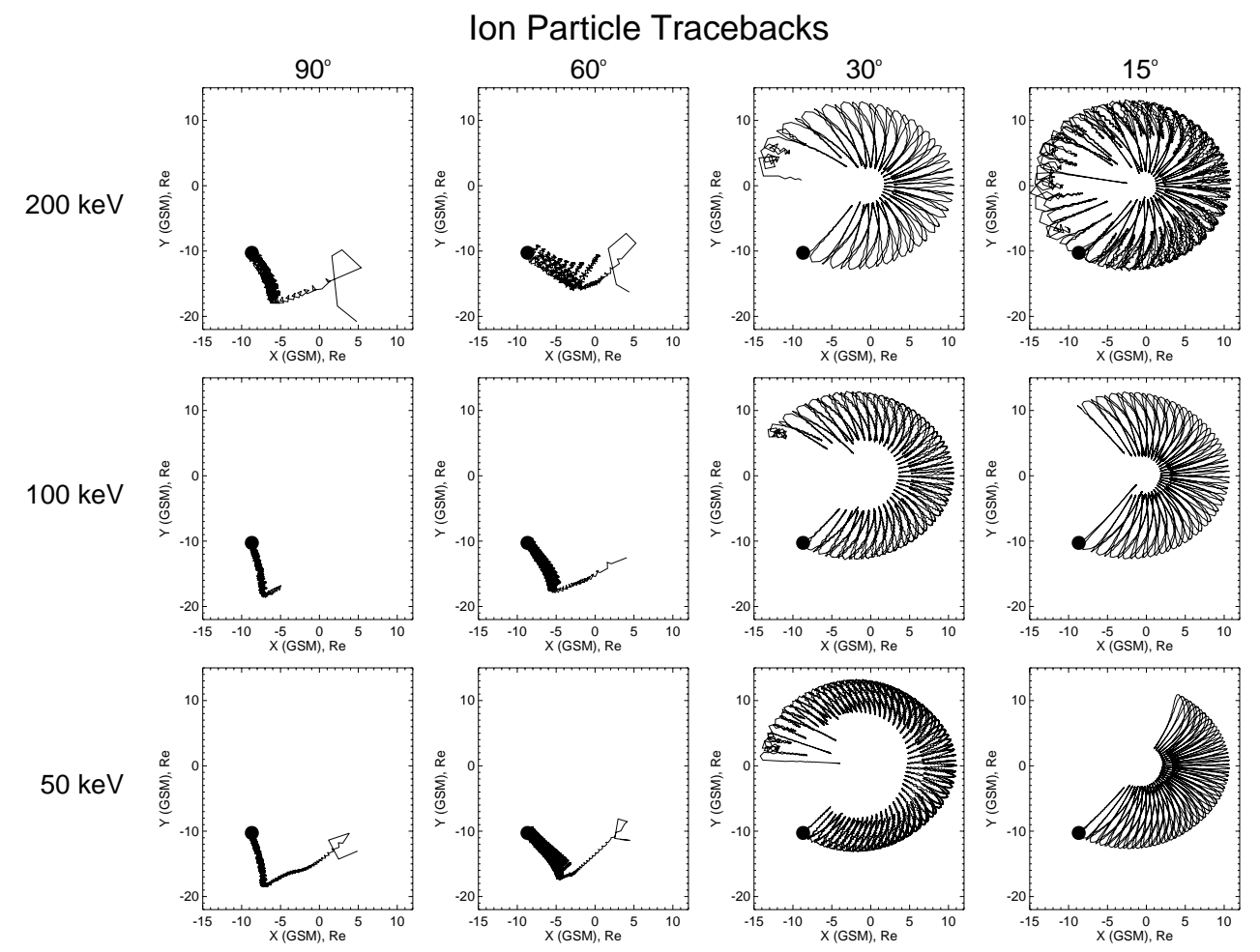

Fig. 13. TRAJGEN trace back of protons from the location of the ISEE-1 satellite at 10:00 UT on 3 March 1979. The trace backs are plotted in the GSM X-Y plane. The protons were plotted for four different pitch angles: $15^{\circ}, 30^{\circ}, 60^{\circ}$, and $90^{\circ}$ and with three different energies: $50 \mathrm{keV}, 100 \mathrm{keV}$, and $200 \mathrm{keV}$.

Figure 13 shows the XY (in GSM) panel obtained from a number of TRAJGEN runs for protons starting at the location of the ISEE-1 satellite at 10:00 UT on 3 March 1979. The protons were given a negative charge in an attempt to try to determine where the high-energy particles are coming from. The code was run for protons with four different pitch angles: $15^{\circ}, 30^{\circ}, 60^{\circ}, 90^{\circ}$ displayed left-to-right in columns from large to small pitch-angle, and with three different energies: 50,100 , and $200 \mathrm{keV}$, displayed in rows in descending energy order. TRAJGEN shows the collective behavior of the particles. For all of the different energies, the large PA $\left(60^{\circ}\right.$ and $\left.90^{\circ}\right)$ particles seem to encounter the morningside magnetopause, suggesting open drift paths. The small PA particles are seen to drift all the way around to the earth's dayside, indicating closed drift paths. Of particular interest is Fig. 14 that plots the same particle trajectories as in Fig. 13 but for the GSM XZ plane. In addition to the open vs. closed drift paths shown in Fig. 13, there is a feature that the protons with small PA pass through the northern high-altitude and high-latitude dayside region (cusp), while those protons with large PA are connected to the high-latitude morningside magnetopause. These runs trace-back a particle to determine its origins. The trace-backs of the different energy protons argue that their source and entry into the plasma sheet is at the dayside northern high-altitude and high-latitude region.

\section{Implications}

\subsection{Drift effects}

A detailed inspection of the top panel in Fig. 4 reveals two effects after 09:14 UT on 3 March 1979. The first effect was the energy dispersion signature that higher energy electron flux was detected earlier. The second effect was the electron fluxes with a PAD peaking at $90^{\circ}$ were observed first at 09:14 UT, then followed by a butterfly PAD after 10:00 UT. The energy dispersion suggests a transport effect from the particle source, since higher energy particles travel faster. The second effect suggests a drift process. Because the large PA charged particles in the closed geomagnetic field lines drift faster than the small PA particles, they will be detected first along the drift path following a particle injection, while the small PA particles will be observed later (e.g., Konradi et al., 1973). The energy dispersion was also observed at 12:00-12:10 UT (see both the valley and peak of the $90^{\circ}$ electron fluxes for different energies in the top panel of Fig. 4). The separation from an isotropic electron PAD started at about 09:14 UT and ended at about 12:10 UT. The above observation thus suggests that from 09:14 UT to 12:10 UT the energetic electrons (E1-E4) measured by ISEE-1 were in a drift process. 


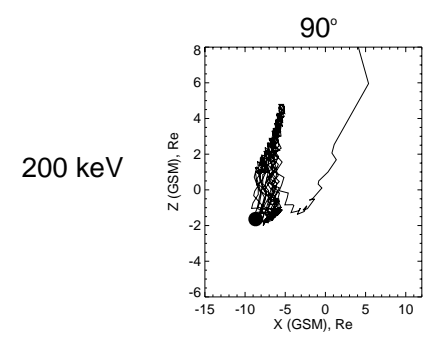

\section{Ion Particle Tracebacks}
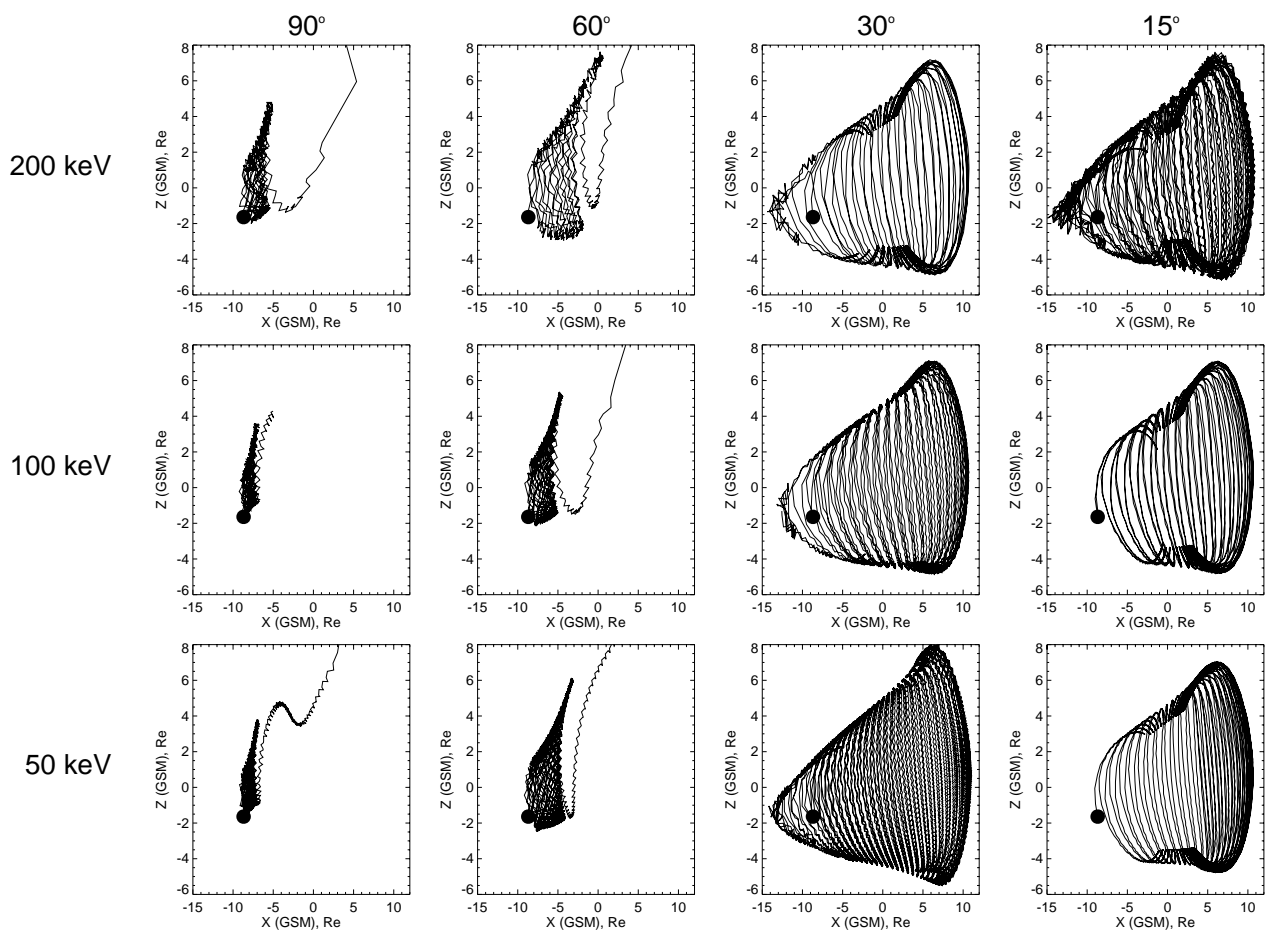

Fig. 14. Similar as in Fig. 13, but plotted in the X-Z plane.

Table 1. The IMF (in GSE) and solar wind pressure on 3 March 1979.

\begin{tabular}{lccccl}
\hline $\mathrm{UT}$ & $\mathrm{Bt}(\mathrm{nT})$ & $\mathrm{Bx}(\mathrm{nT})$ & $\mathrm{By}(\mathrm{nT})$ & $\mathrm{Bz}(\mathrm{nT})$ & Pressure(nPa) \\
\hline $04: 00$ & 6.5 & 5.7 & -2.0 & 2.4 & 1.36 \\
$05: 00$ & 6.4 & 4.5 & -2.5 & 1.0 & 1.47 \\
$06: 00$ & 6.4 & 2.6 & -5.3 & -1.6 & 1.73 \\
$07: 00$ & 6.3 & 4.2 & -4.1 & 1.4 & 1.62 \\
$08: 00$ & 6.2 & 4.1 & -3.7 & 1.1 & 1.68 \\
$09: 00$ & 6.6 & 5.2 & -2.2 & 3.1 & 1.55 \\
$10: 00$ & 6.7 & 5.4 & -1.3 & 3.4 & 1.59 \\
$11: 00$ & 6.8 & 4.3 & -0.5 & 4.7 & 1.69 \\
$12: 00$ & 6.5 & 1.8 & -5.0 & -2.3 & 1.77 \\
\hline
\end{tabular}

The case is different for energetic protons measured by ISEE-1. In Fig. 6, the separation from an isotropic proton PAD started at about 08:30 UT for P1-P3, and at about 09:00 UT for P4, earlier than for the electrons. There were two additional different features at 08:30-09:00 UT: (1) no obvious energy dispersion and (2) no peak at $90^{\circ} \mathrm{PAD}$ were observed except for P3-P4 at about 8:35 UT when the peak flux of the $90^{\circ} \mathrm{PA}$ protons was detected earlier than that of the P1-P2 large PA protons. The energy dispersion signature was observed only at 12:00-12:10 UT for small PA proton fluxes. Compared with the electron drift signatures (Fig. 4) the proton observations with no obvious energy dis- persion (Fig. 6) imply that the drift paths that connected to the charged particle source were much shorter than those of electrons measured by ISEE-1. In other words, the protons at ISEE-1 location were much closer to the particle source than electrons along their trajectoties.

On the other hand, both Figs. 4 and 6 show that around 08:30 to 09:00 UT on 3 March 1979, ISEE-1 detected a proton population that has been affected by encountering the magnetopause as it was drifting toward the spacecraft. Due to drift directions the magnetopause was closer for the proton population than it was for electrons. Shortly after that (about 09:30-10:00 UT) electrons showed activity that had a signature of injection followed by both energy and pitch angle dispersion. Both the injection and the depth of the butterflys are energy dependent. The varying depth of the butterflys may be in part due to an energy dependent radial gradient in the electron population acting as a source for the ISEE observations. Of interest is the fact that both the electron (top panel of Fig. 4) and proton (Fig. 6) populations return to their previous isotropic distributions at basically the same time, about 12:15 UT, suggesting the crossing of a particle boundary of some kind. Table 1 lists the hourly interplanetary magnetic field (IMF) data in geocentric solar ecliptic (GSE) coordinates and the solar wind pressure from the OMNI data set. It shows that there were two southward turnings in the IMF at 06:00 UT and 12:00 UT on 3 March, 1979, each may usher in substorm activity. The AE index (bottom panel of Fig. 4) 
showed an abrupt increase at about 11:50 UT. It increased from about $60 \mathrm{nT}$ to $120 \mathrm{nT}$ in 5-7 min, (not substorm activity, but geomagnetic activity), about $10 \mathrm{~min}$ before the butterfly PADs disappear completely. Since both the ISEE-1 trajectory and the local magnetic field did not change drastically at 11:50 UT, the increase in the AE may probably indicate a change in the magnetospheric configuration and disconnected ISEE-1 from the particle source, thus ending the butterfly PADs.

\subsection{A new particle source}

Magnetopause shadowing with magnetic field drift shell splitting have been used to explain the butterfly PADs before (e.g., West et al., 1972, 1973). At the location of ISEE-1 during the period of interest, the $90^{\circ} \mathrm{PA}$ charged particles near the geomagnetic equator were more likely on open drift paths than small PA particles to intercept the magnetopause and were lost to the magnetosheath. Since this mechanism is independent of particle energy, the butterfly shape (the difference in flux between small PA and large PA particles) should be similar if there is no new particle source. However, the top panel of Fig. 4 shows that the difference in flux between small PA and large PA electrons at 09:00-12:00 UT for E1 was much less than that for $\mathrm{E} 3$ and $\mathrm{E} 4$, suggesting there was a new particle source to supply continually the E1 (also E2) $90^{\circ}$ electrons at the time. Furthermore, since the local magnetic field measured by ISEE-1 was almost constant during 07:00-12:00 UT, the $90^{\circ} \mathrm{PA}$ charged particle (both proton and electron) intensities over the same energy range should be very close between 07:30 UT and 09:00-12:00 UT if there was no new source of particles. The measured higher fluxes for P1-P4 (Fig. 6) and for E1-E2 (Fig. 4) at 09:00-12:00 UT also suggest a new particle source.

This new particle source is not the substorm. In addition to the AE index being below 100 nT for the period 09:1011:50 UT (40 nT at 10:00 UT) (see bottom panel of Fig. 4), the LANL geosynchronous satellites observed no substorms (no particle injections). It is really surprising that ISEE-1 measured up to three orders of magnitude enhancements of the energetic charged particles, while no substorms were observed at geostationary altitudes. The charged particle drift effects discussed in last subsection also argue against a nightside particle source within the local time interval from 18:00 MLT to 03:00 MLT. The particle trajectory tracings shown in Sect. 4 suggest that this new particle source is located in the northern high-altitude and high-latitude (Fig. 14) morningside (Fig. 13) region, which is consistent with the drift effect observations aforementioned.

\subsection{Cusp source}

In 1996, MeV charged particles were detected in the dayside high-altitude cusp region (Chen et al., 1997, 1998; Chen and Fritz, 1998; Sheldon et al., 1998). These cusp energetic particles (CEP) with energies from $20 \mathrm{keV}$ up to $10 \mathrm{MeV}$ were found to be associated with large diamagnetic cavities and strong magnetic field turbulence. These cusp diamagnetic cavities are always there day after day and are as large as $6 R_{E}$ under normal solar wind conditions (Fritz et al., 2003b). The intensities of the CEPs have been observed to increase by as much as four orders of the magnitude when compared with regions adjacent to the cusp, indicating that the high-altitude dayside cusps (both northern and southern) are extremely dynamic regions in geospace (Chen and Fritz, 2005; Chen et al., 2005). The cusp magnetic field lines are connected with the entire magnetopause boundary layers, so that the CEPs, as a new particle source, may transport particles into the magnetopause, form an energetic particle layer there, and contribute to the nightside particle populations through charged particle drifting (Fritz and Chen 1999; Chen and Fritz, 2002).

The connection between the cusp and the nightside geomagnetic tail has been suggested long ago based upon two off-equatorial field minima in the high-altitude dayside cusps caused by solar wind pressure (Mead, 1964; Shabansky and Antonova, 1968; Shabansky, 1971). The connection between cusp particles and nightside particles through their drift paths has been showed by numerical simulations using different tracing codes (Delcourt and Sauvaud, 1999; Blake, 1999; Fritz et al., 2000; Antonova et al., 2000).

Table 1 indicates that from 07:00 UT to 12:00 UT on 3 March 1979, the IMF had a negative $B_{y}$ (dawnward) component. According to the prediction of the current MHD models a negative IMF $B_{y}$ would move the dayside northern cusp dawnward into morningside (e.g., Cowley et al., 1991; Crooker et al., 1998). Recent in situ satellite observations indicate that almost all POLAR cusp crossiongs were identified as CEP events (Fritz et al., 2003b; Chen and Fritz, 2005). Since ions drift westward and electrons drift eastward, the protons measured by ISEE-1 at 03:00 MLT would be much closer to the morningside particle source than that of electrons. Therefore, a cusp particle source in the northern highaltitude and high-latitude morningside agrees with both the drift effect observations and the particle trajectory tracings.

\section{Conclusions}

From 07:00 UT to 14:00 UT on 3 March 1979, both ISEE satellites were inbound from beyond $15 R_{E}$ to about $10 R_{E}$ at 03:00 MLT, and observed unusual energetic particle PADs. Our principal conclusions are the following:

(1) ISEE-1 observed a butterfly PAD for a period of about $2 \mathrm{~h}(10: 00-12: 00 \mathrm{UT})$ for the electrons, and $3 \mathrm{~h}(09: 00-$ 12:00 UT) for the protons.

(2) The small PA $\left(15^{\circ}, 30^{\circ}\right)$ charged particles (electrons and protons) were seen to behave collectively in all four energy ranges. The relative differences in electron fluxes 
between $15^{\circ} \mathrm{PA}$ and $90^{\circ} \mathrm{PA}$ were more significant for higher energy channels during the butterfly PAD period (about 10:00-12:00 UT).

(3) Three different types of electron PADs (butterfly, isotropic, and peaked-at- $90^{\circ}$ ) were observed at the same location and time as a function of energy.

(4) Electron butterfly PADs were also observed by the ISEE-2 for about $1.5 \mathrm{~h}$, although less well resolved than ISEE-1. Unlike the ISEE-1, no butterfly distributions were resolved in the ISEE-2 proton PADs due to less angular resolution of the ISEE-2 energetic particle instrument.

(5) More obvious drift effects were measured by ISEE-1 at 03:00 MLT for electrons than for the protons, suggesting that the measured electrons were much further away than the protons from the particle source along their drift paths. This ruled out a nightside source within 18:00 MLT to 03:00 MLT.

(6) No obvious substorms were detected during the period 09:10-11:50 UT.

(7) The results (5) and (6) reveal that the measured charged particle fluxes were due to a new particle source other than the substorm source.

(8) Particle trajectory tracings show that the $50-200 \mathrm{keV}$ protons with small PA at the ISEE-1 location at 10:00 UT on 3 March 1979 were passing through the northern high-altitude and high-latitude morningside region, while those protons with large PA were connected to the high-latitude morningside magnetopause.

(9) It is possible that the cusp particle source is responsible for the all particles observed during the event.

Acknowledgements. We thank both referees for their very helpful, detailed, and important comments and suggestions. We would like to knowledge the efforts and discussions with J. D. Sullivan of Boston University regarding the particle tracing code, TRAJGEN. We also thank M. M. Klida and N. M. Lender for assistance in ploting Figs. 4 and 10. The work of D. L. Matthews in the recovery of the ISEE-1 and 2 energetic particle data and the Principal Investigators, D. J. Williams of the Medium Energy Particle Experiment (MEPE) and C. T. Russell of the magnetic field experiment (MFE) are also gratefully acknowledged along with the Institute of Geophysics and Planetary Physics at UCLA for providing the magnetic field data collected by ISEE-1 and ISEE-2. The Satellite Situation Center Website (SSCWeb) for the satellite's trajectory during the time of the event is acknowledged also.

Topical Editor T. Pulkkinen thanks two referees for their help in evaluating this paper.

\section{References}

Antonova, A. E., Gubar, Yu. I., and Kropotlin, A. P.: Energetic particle population in the high-latitude geomagnetosphere, Phys. Chem. Earth (C), 25(1-2), 47-50, 2000.

Blake, J. B.: Comment on "Cusp: A new acceleration region of the magnetosphere" by J. Chen et al., Czech. J. Phys., 49(4a), 675-677, 1999.

Chen, J. and Fritz, T. A.: Correlation of cusp MeV helium with turbulent ULF power spectra and its implications. Geophys. Res. Lett., 25, 4113-4116, 1998.

Chen, J. and Fritz, T. A.: The global significance of the CEP events, in Solar-Terrestrial Magnetic Activity and Space Environment, edited by: Wang, H. N. and Xu, R. L., COSPAR Colloquia Series, 14, 239-249, 2002.

Chen, J. and Fritz, T. A.: High-altitude cusp: The extremely dynamic region in geospace, in The Magnetospheric Cusps: Structure and Dynamics, edited by: Fritz, T. A. and Fung, S. F., Surveys in Geophysics, 26:1-3, 71-93, 2005.

Chen, J., Fritz, T. A., and Sheldon, R. B.: Comparison of energetic ions in cusp and outer radiation belt, J. Geophys. Res., 110, A12219, doi:10.1029/2004JA010718, 2005.

Chen, J., Fritz, T. A., Sheldon, R. B., et al.: A new, temporarily confined population in the polar cap during the August 27, 1996 geomagnetic field distortion period. Geophys. Res. Lett., 24, 14471450, 1997.

Chen, J., Fritz, T. A., Sheldon, R. B., et al.: Cusp energetic particle events: Implications for a major acceleration region of the magnetosphere, J. Geophys. Res., 103, 69-78, 1998.

Cowley, S. W. H., Morelli, J. P., and Lockwood, M.: Dependence of convective flows and particle precipitation in the high-latitude dayside ionosphere and the $\mathrm{X}$ and $\mathrm{Y}$ components of the interplanetary magnetic field, J. Geophys. Res., 96, 5557-5564, 1991.

Crooker, N. U., Lyon, J. G., and Fedder, J. A.: MHD model merging with IMF By: Lobe cells, sunward polar cap convection, and overdraped lobes, J. Geophys. Res., 103, 9143-9152, 1998.

Delcourt, D. C. and Sauvaud, J.-A.: Populating of the cusp and boundary layers by energetic (hundreds of $\mathrm{keV}$ ) equatorial particles, J. Geophys. Res., 104, 22 635-22 648, 1999.

Eccles, A. A. and Fritz, T. A.: Energetic particle observations at the subsolar magnetopause, Ann. Geophys., 20, 445-460, 2002, http://www.ann-geophys.net/20/445/2002/.

Fritz, T. A. and Chen, J.: The cusp as a source of magnetospheric particles, Radiation Measurements, 30(5), 599-608, 1999.

Fritz, T. A., Chen, J., Sullivan, J. D., and Karra, M.: Is the cusp a source or a sink for magnetospheric energetic particles?, Proc. Int. Symposium: From solar corona through interplanetary space, into Earth's magnetosphere and ionosphere: Interball, ISTP satellites, and ground-based observations, (Kyiv, Ukraine), 205-209, 2000.

Fritz, T. A., Alothman, M., Bhattacharjya, J., Matthews, D. L., and Chen, J.: Butterfly Pitch-angle Distributions Observed by ISEE-1, Planetary and Space Sciences, 51, 205-219, 2003a.

Fritz, T. A., Chen, J., and Siscoe, G. L.: Energetic ions, large diamagnetic cavities, and Chapman-Ferraro cusp, J. Geophys. Res., 108(A1), 1028-1036, 2003b.

Konradi, A., Williams, D. J., and Fritz, T. A.: Energy spectra and pitch angle distributions of storm-time and substorm injected protons, J. Geophys. Res., 80, 543-552, 1975.

Kosik, J. C.: Influence of electric field on charged particle mo- 
tion and electron fluxes at synchronous altitudes, in Quantitative Modeling of Magnetospheric Processes, edited by: Olson, W. P., Geophys. Monogr. Series, 21, 569-581, 1979.

Mead, G. D.: Deformation of geomagnetic field by the solar wind, J. Geophys. Res., 69, 1181-1195, 1964.

McIlwain, C. E.: Coordinates for mapping the distribution of magnetically trapped particles, J. Geophys. Res., 66, 3681-3691, 1961.

McIlwain, C. E.: Substorm injection boundaries in Magnetospheric Physics, edited by: McCormac, B. M., D. Reidel, Dordrecht, Holland, 1974.

Pfitzer, K., Lezniak, T. W., and Winckler, J. R.: Experimental verification of drift shell splitting in the distorted magnetosphere, J. Geophys. Res., 74, 4687-4693, 1969.

Reeves, G. D., R. D. Belian, R. D., and Fritz, T. A.: Numerical tracing of energetic particle drifts in a model magnetosphere, J. Geophys. Res., 96, 13 997-14 008, 1991.

Roederer, J. G.: On the adiabatic motion of energetic particles in a model magnetosphere, J. Geophys. Res., 72, 981-992, 1967.

Roederer, J. G.: Dynamics of Geomagnetically Trapped Radiation, Springer-Verlag, New York, 23-28, 1970.

Russell, C. T.: The ISEE1 and 2 fluxgate magnetometers, IEEE Trans. Geosci. Electr., GE-16, 239-242, 1978.

Shabansky, V. P.: Some processes in the magnetosphere, Space Sci. Rev., 12, 299-418, 1971.

Shabansky, V. P. and Antonova, A. E.: Topology of particle drift shells in the Earth's magnetosphere, Geomangn. Aeron., 8, $993-$ 997, 1968.

Sheldon, R. B., Spence, H. E., Sullivan, J. D., Fritz, T. A., and Chen, J.: The discovery of trapped energetic electrons in the outer cusp, Geophys. Res. Lett., 25, 1825-1828, 1998.

Sibeck, D. G., McEntire, R. W., Lui, A. T. Y., Lopez, R. E., and Krimigis, S. M.: Magnetic field drift shell splitting: Cause of unusual dayside particle pitch angle distributions during storms and substorms, J. Geophys. Res., 92, 13 485-13 497, 1987.
Smets, R., Delcourt, D., Sauvard, J. A., and Koperski, P.: Electron pitch angle distributions following the dipolarization phase of a substorm: Interball-Tail observations and modeling. J. Geophys. Res. 104, 14 571-14 581, 1999.

Sullivan, J. D.: Hunstsville 2000 conference proceedings CDROM Software Section Models: TRAJGEN at http://science.msfc. nasa.gov/ssl/pad/sppb/workshop7/html/cd.htm, 2002.

Tsyganenko, N. A. and Usmanov, A. V.: Determination of the magnetospheric current system parameters and development of experimental geomagnetic field models based on data from IMP and HEOS satellites, Planet. Space Sci., 30, 985-998, 1982.

Tsyganenko, N. A.: Modeling the Earth's magnetospheric magnetic field confined within a realistic magnetopause. J. Geophys. Res., 100, 5599-5612, 1996.

Tsyganenko, N. A. and Fairfield, D. H.: Global shape of the magnetotail current sheet as derived from Geotail and Polar data, J. Geophys. Res., 109, A03218, doi:10.1029/2003JA010062, 2004.

West Jr., H. I.: Some Observations of the trapped electrons produced by the Russian high altitude nuclear detonation of October 28, 1962, Radiation Trapped in the Earth's Magnetic Field, edited by: McCormac, B. M., D. Reidel Publishing Company, Dordrecht, Holland, 634, 1965.

West Jr., H. I., Buck, R. M., and Walton, J. R.: Shadowing of electron azimuthal drift motions near the noon magnetopause, Nature Phys. Sci., 240, 6-7, 1972.

West Jr., H. I., Buck, R. M., and Walton, J. R.: Electron pitch angle distributions of energetic electrons throughout the magnetosphere as observed by OGO-5. J. Geophys. Res. 78, 1064-1081, 1973.

Williams, D. J., Keppler, E., Fritz, T. A., Wilken, B., and Wibberenz, G. W.: The ISEE-1 and 2 medium energy particles experiment, IEEE Trans. Geosci. Electr., GE-16, 270-278, 1978. 